Review Articles

\title{
A Perspective on 3D Bioprinting Technology: Present and Future
}

\author{
Shweta Agarwala \\ Singapore center for $3 D$ Printing, \\ Mechanical and Aerospace Engineering, Nanyang Technological University, Singapore
}

Article history

Received: 03-11-2016

Revised: 05-11-2016

Accepted: 09-11-2016

Email: agarwala.shweta@gmail.com

\begin{abstract}
Bioprinting, an additive manufacturing technique, has become the cynosure of research and industry world alike. This emerging technology holds the promise of constructing artificial tissues and organs in future. This paper overviews the current state in bioprinting technology, distinguishes between different types of techniques and describes the bio-inks. Current challenges and limitations inhibiting the growth of this field are also discussed.
\end{abstract}

Keywords: Bioprinting, Additive Manufacturing, 3D Printing, Biofabrication, Bio-Inks

\section{Introduction}

Last decade has seen rapid rise in the field of threedimensional (3D) printing, also known as additive manufacturing (Agarwala and Yeong, 2016; Chua et al., 2003; Goh et al., 2016; Kruth et al., 1998). The immense interest in the technology comes from the fact that structures can be built bottom-up easily, fast, costeffectively and in customized manner according to the end user. Most importantly, 3D printing can create intricate designs and complex structures with ease using CAD models (Chua et al., 2003). The customizable and easy processing of $3 \mathrm{D}$ printing has made it an integral tool in rapid prototyping for both industry and research and with applications ranging from home décor, furniture and toy to aerospace and military.

There has been particular interest in using 3D printing capability for bio-medical field. Although last decade has already seen rise in 3D printed bio-medical devices, appliances and accessories (Giannatsis and Dedoussis, 2009; Lee et al., 2016; Yeong and Chua, 2013), the field is now moving on to print live cells, tissues and even organs (Murphy and Atala, 2014; Mironov et al., 2013). Bioprinting, as it is commonly referred, holds the promise to print standalone or embedded cells on any surface in a customized manner for added functionality. Bioprinting is especially attractive to build tissue model platforms and for tissue engineering field. Traditional approach towards tissue models and engineering is to seed cells on to a porous and mechanically stable scaffold. However, such approach suffers from drawbacks like limited or no control on cell placement, limited cell density, unable to fabricate complex scaffold shapes etc. Hence, new bio-fabrication approaches are critical to generate 2D and $3 \mathrm{D}$ complex biological constructs using living cells and engineered biomaterials. Bioprinting may overcome issues faced by traditional fabrication processes and provide required accuracy, flexibility, viability and reproducibility for biomedical applications (Wang et al., 2015).

Bioprinting directly writes the living cells and biomaterials in a layer-by-layer fashion utilizing the initial CAD model of the final object or design. Hence, bioprinting can allow precise position capability that is required to create tissue model mimicking real human body structures with high cell density. The added in-situ skill can help to realize the dream of printing real body parts to solve the shortage of transplantable organs. This paper provides a general overview of the current state of bioprinting, elaborating on different technologies- laser, extrusion and inkjet. The paper summarizes printing strategies, current state and materials for bioprinting. Finally, challenges of the field and future research and trends are discussed.

\section{Bioprinting Method}

$3 \mathrm{D}$ biopriting is heralded as the next big revolution in biomedical and biotechnology field. Like other additive manufacturing techniques; namely fused deposition modeling, stereolithography, digital light processing, selective laser sintering and selective laser melting; the $3 \mathrm{D}$ bioprinting also works on building the end product from a computer model in layer-by-layer fashion. The 
model file could be designed using several ways like Computer-Aided Design (CAD), Computer Tomography (CT) scanning or Magnetic Resonance Imaging (MRI) and used to print on the required printer (Hutmacher et al., 2004; Peltola et al., 2008; Seol et al., 2012). The bioprinting techniques either fall under orifice based or orifice-free methods, which can be further classified in to four main categories depending on their method of operation. Figure 1 shows the schematic diagrams depicting various types of bioprinting techniques.

\section{Inkjet Based}

An inkjet-based printer is a non-contact printing technique that places extremely small droplets of ink on the substrate (Xu et al., 2013; Abeyewickreme et al., 2009; Fang et al., 2012). Inkjet printing could be further divided in to continuous or drop-on-demand methodology to create cellular constructs in a desired manner. Continuous inkjet technique produces small droplets of ink from a reservoir through a nozzle in to a continuous and steady stream via instability mechanism (Xu et al., 2013). The ink droplets are subjected to an electrostatic field and drops are deflected to print on the substrate or remain undeflected to be re-used. Drop-on-demand, as the name suggests, produces drops at regular intervals as and when required (Binder et al., 2011). It can be classified in to thermal type or piezoelectric type. In thermal inkjet printing, a heating element helps to generate a bubble and eject the small droplet from the nozzle. A piezoelectric material, controlled by voltage, is actuated and dispenses the ink droplets.

\section{Extrusion Based}

Extrusion is a technique where continuous filaments of a material are forced through a nozzle in a controlled manner to construct a $3 \mathrm{D}$ structure. There is no droplet generation; instead a continuous stream of bio-inks is dispensed on the substrate. The printer setup normally consists of an orifice made of syringes, a motor that drives the syringes in 3 -axis motion and a stage on which the object is constructed (Fang et al., 2012). For extrusion printing of cells, the material usually consists of a highly viscous cell-laden hydrogel that can flow from the nozzle without the need for high temperatures (Fedorovich et al., 2007).

\section{Microvalve Based}

The deposition of living cells using microvalve dispensing systems was first explored by Demirci and Montesano (2007), who developed a custom printing tool where four of these dispensers were mounted above a three-axis robotic stage for use in cell printing. Microvalve printing makes use of constant pneumatic pressure to dispense small droplets through a valve. A controlled pressure system will force the bio-ink to go through the nozzle. The valve can operate under mechanical, electrical and magnetic influence to open and close. The nozzle size is usually in 100-300 $\mu \mathrm{m}$ in diameter. Different pressure control systems are used to print bio-inks of varying viscosities, as each may require different level of control. Microvalve-based bioprinting methods have high printing speed $\left(10-50 \mu \mathrm{m} \mathrm{sec}^{-1}\right)$ and high cell density.

\section{Laser Based}

Laser based bioprinting is also called orifice free printing where a laser guides the writing process. The setup consists of a laser beam source, focusing system and stage. The normal mode of operation involves focusing a laser beam to create a light-trap, which is used to guide the cells on the substrate (Malda et al., 2013). Another variation of this system, laser-induced forward transfer (LIFT) is more commonly used for printing cells (Bohandy et al., 1986; Odde and Renn, 2000; Schiele et al., 2010). Here the focused laser causes local vaporization of energy absorbing layer and the generated droplets are received by the substrate facing the bio-ink or biomaterials.
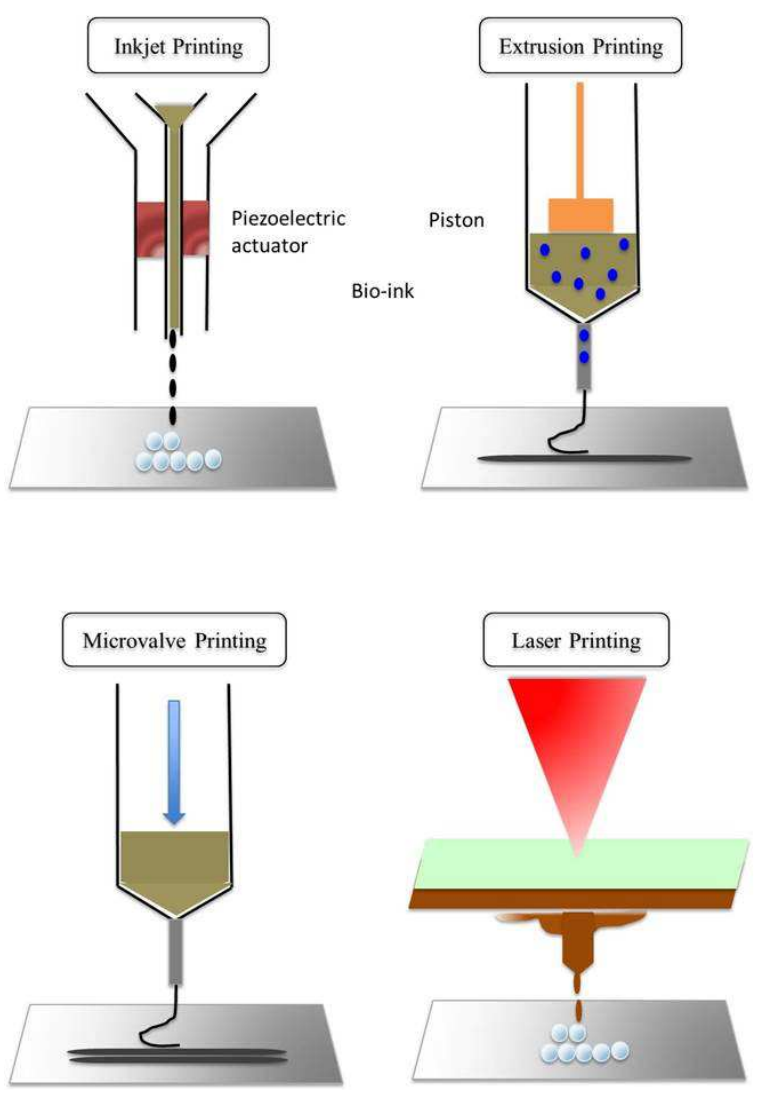

Fig. 1. Schematic showing various bioprinting techniques used 


\section{Bio-Inks and Biomaterials}

The formulation of right bio-ink to get the desired properties and geometry remains the crucial aspect of bioprinting technique. A lot of applications require bioinks to be laden with cells and this requires the formulations to have certain properties. The inks needs to fulfill biological, mechanical and physical attributes to be printed with cells. Thus, it is important to be aware of the desired characteristics in order to synthesize appropriate bio-ink for various bioprinting techniques.

\section{Hydrogels}

Hydrogels are class of materials that are mostly composed of water. They are water insoluble, crosslinked, 3D networks of polymer chains and water. The water mainly fills the voids between polymer chains. Crosslinking facilitates insolubility in water and provides required mechanical strength and physical integrity. Properties like biocompatibility, ability to protect cells, good nutrient transport and in-vivo functions have made hydrogels the star biomaterial. These features also make them ideal candidates to act as Extracellular Matrix (ECM) component and allow cell encapsulation in 3D environment (Fedorovich et al., 2007; Pati et al., 2014). Hydrogels can be classified in variety of ways- depending on cross-linking, physical or chemical bond or physical structure of polymer chains. Another way to categorize these novel materials is on basis of how they are derived- natural or synthetic. The former hydrogels are fully biocompatible and biodegradable (Hunt et al., 2010; Patterson et al., 2010; Spiller et al., 2011), unlike latter that may contain some toxic substances and may lack in some bioactive properties. However one big advantage of synthetic hydrogels is that they can be precisely tailored to give wide range of properties (Censi et al., 2011; Dash et al., 2011). Examples of hydrogels that have natural origin are proteins like collagen and polysaccharides like chitosan, dextran and alginate. Synthetic hydrogels are made from monomers such as vinyl acetate, acrylamide, ethylene glycol and lactic acid. In bioprinting, hydrogels are used as bio-ink materials or as cell delivery vehicles. Many types of cells have been encapsulated within hydrogels and tested, such as fibroblasts, chondrocytes, hepatocytes, smooth muscle cells, adipocytes and stem cells (Lutolf et al., 2009; Savas and Utkan, 2013).

\section{Cell Printing}

Crucial for the application of cell printing is a high survival rate and no damage to the genotype and phenotype of the cells. Cell-aggregate-based bio-inks can be homogeneous, containing a single-cell type, or heterogeneous, prepared by coculturing several cell types. The cell-aggregate-based bio-inks typically used are tissue spheroids, cell pellets and tissue strands. In addition to hydrogels and cell aggregate-based bio-inks, decellularized matrix components have been recently considered as a new bio-ink type. Dong-Woo Cho's group used decellularized adipose, heart and cartilage tissue matrix components and printed with Polycaprolactone (PCL) framework to support tissue analogues in 3D (Pati et al., 2014).

\section{Scaffolds and Constructs}

Scaffolds and constructs form the backbone of tissue engineering, as they provide the necessary framework to support cell attachment and tissue regeneration. Printing of scaffolds and constructs laden with cells and materials is most common type of biofabrication carried out. In bioprinting, a substrate is usually used as a bioprocessable scaffolding material, contributing as a biological and structural support for cells to attach, proliferate and differentiate. Biocompatible, nontoxicity, dispensability, rapid solidification and functionality with growth factors for high cell viability are some properties required for the scaffolds. Thus far, various hydrogels such as collagen and soy agar gel have been used as a substrate material (John et al., 1977; Lee and Yeong, 2016; Savas and Utkan, 2013). The traditional method of scaffold fabrication uses "mold and fill" method, which is replete with limitations. Controlling the placement of cells on scaffolds precisely, having controlled architecture and degradation of material with time are some problems that the traditional method is unable to address (Yeong et al., 2004). Bioprinting may overcome some of these limitations.

\section{D Printing}

A recent advancement in $3 \mathrm{D}$ printing has led to evolution of $4 \mathrm{D}$ printing. $4 \mathrm{D}$ printing is loosely defined when another dimension time is added to $3 \mathrm{D}$ printing. 4D bioprinting can be considered a promising direction in the fabrication of living cells in a shorter period of in vitro culture time (Gao et al., 2016). It enables the user to design almost any arbitrary shape, transformable to another shape using wide variety of materials. The arena of $4 \mathrm{D}$ printing has opened up a new platform where different properties can be combined to add functionalities in existing systems and thus have dynamic microstructures that can have wide applications (Jamal et al., 2013). For bio-medical field, 4D printing can enable capabilities where objects constructed using bio-inks can fuse, fold and re-model to generate tissues and constructs in fourth dimension of time. This can help mimic real life body functions and mechanisms. The degree of recovery, folding angle and tenability will depend on the properties of the materials, cell behavior, growth media and other parameters. 


\section{Challenges}

The choice of printing technique to be used will depend on lot of factors like complexity of design, desired geometry, nature and volume of bio-ink. The field of bioprinting is still in its infancy and requires a lot of challenges to be answered. There is lot of room for improvement in printers where nozzles and print heads can be designed for finer resolution, multimaterial printing, higher degree of freedom and more automated control.

Vascularization of engineered tissues is a key issue that needs to be fully addressed for the bio-medical field to be successful. This is especially true for $3 \mathrm{D}$ tissue constructs requiring adequate supply of oxygen and nutrients. To take a step further, it would be great if bio-inks can incorporate well-connected capillaries to realize functional tissues and printed organs. This will also require bio-inks and biomaterials to be developed in a special way to have multicellular aggregates for self-vascularization. Material innovation is also needed to impart sufficient mechanical strength after printing, so that structures are able to retain their shape. Extrusion and microvalve based printing suffers from a common problemlack of mechanical integrity in the printed hydrogel constructs. This has not only limited the scale and fidelity of the constructs but has also posed challenges for implantation of the printed structure in vivo. Some research groups have worked towards solving the issue. Recently, Schuurman et al. (2011) employed extrusion printing of soft, cell-laden hydrogel with another stiffer synthetic polymer. The group demonstrated sequential printing of Polycaprolactone (PCL) and cell-laden alginate, where alginate component was crosslinked with calcium chloride following deposition.

Scalability, reliability and repeatability are others concerns that need to be addressed for 3D printing technologies. With the world talking about production of organs, the above features become even more important.

\section{Conclusion}

This review paper provides a general overview of the current state of the art of bioprinting technology. A distinction between laser-, extrusion- and inkjetbased bioprinting technologies is made. Various types of bio-inks are also discussed. Even though the biofabrication of whole organs has not yet been achieved, bioprinting is already changing the way tissues and organs are studied by providing reliable constructs with increasing complexity and resemblance to native tissues. However, despite the great progress and breakthroughs, bioprinting technology is still in its infancy and needs to overcome several challenges and limitations.

\section{Ethics}

This article is original. Authors declare no ethical issues that may arise after the publication of this manuscript.

\section{References}

Abeyewickreme, A., A. Kwok, J.R. McEwan and S.N. Jayasinghe, 2009. Bio-electrospraying embryonic stem cells: Interrogating cellular viability and pluripotency. Integrative Biol., 1: 260-266. DOI: $10.1039 / \mathrm{b} 819889 \mathrm{f}$

Agarwala, S. and W.Y. Yeong, 2016. 3D printed electronics tracks for bio-integrated free-form devies. Proceedings of the 2nd International Conference on Progress in Additive Manufacturing, May 16-19, Chua C-K, Ed. Singapore, pp: 313-316.

Binder, K.W., A.J. Allen, J.J. Yoo and A. Atala, 2011. Drop-on-demand inkjet bioprinting: A Primer. Gene Therapy Regulat., 6: 33-49. DOI: $10.1142 / \mathrm{S} 1568558611000258$.

Bohandy, J., B.F. Kim and F.J. Adrian, 1986. Metal deposition from a supported metal film using an excimer laser. J. Applied Phys., 60: 1538-1539. DOI: $10.1063 / 1.337287$

Censi, R.S.W., J. Malda, G. di Dato, P. Burgisser and W. Dhert et al., 2011. A printable photopolymerizable thermosensitive p(HPMAmlactate)-PEG hydrogel for tissue engineering. Adv. Funct. Mater., 21: 1833-1842. DOI: $10.1002 /$ adfm.201002428.

Chua, C.K., K.F. Leong and C.S. Lim, 2003. Rapid Prototyping: Principles and Applications. 2nd Edn., World Scientific.

Dash, M., F. Chiellini, R. Ottenbrite and E. Chiellini, 2011. Chitosan-a versatile semi-synthetic polymer in biomedical applications. Prog. Polym. Sci., 36: 981-1014.

DOI: 10.1016/j.progpolymsci.2011.02.001

Demirci, U. and G. Montesano, 2007. Cell encapsulating droplet vitrification. Lab Chip, 7: 1428-1433. DOI: 10.1039/b705809h

Fang, Y., J.P. Frampton, S. Raghavan, R. Sabahi-Kaviani and G. Luker et al., 2012. Rapid generation of multiplexed cell cocultures using acoustic droplet ejection followed by aqueous two-phase exclusion patterning. Tissue Eng. Part C: Meth., 18: 647-657. DOI: $10.1089 /$ ten.TEC.2011.0709

Fedorovich, N.E., J. Alblas, J.R.D.E. Wijn, W.E. Hennink and A.J. Verbout et al., 2007. Hydrogels as extracellular matrices for skeletal tissue engineering: State-of-the-art and novel application in organ printing. Tissue Eng., 13: 1905-1925. DOI: $10.1089 /$ ten.2006.0175. 
Gao, B., Q. Yang, X. Zhao, G. Jin and Y. Ma et al., 2016. 4D bioprinting for biomedical applications. Trends Biotechnol., 34: 746-756. DOI: $10.1016 /$ j.tibtech.2016.03.004

Giannatsis, J. and V. Dedoussis, 2009. Additive fabrication technologies applied to medicine and health care: A review. Int. J. Adv. Manufact. Technol., 40: 116-127. DOI: $10.1007 / \mathrm{s} 00170-007-1308-1$

Goh, G.L., J. Ma, K.L.F. Chua, S. Agarwala and W.Y. Yeong et al., 2016. Inkjet-printed patch antenna emitter for wireless communication application. Virtual Phys. Prototyp. DOI: 10.1080/17452759.2016.1229802

Hunt, N. and L. Grover, 2010. Cell encapsulation using biopolymer gels for regenerative medicine. Biotechnol. Lett., 32: 733-742.

DOI: $10.1007 / \mathrm{s} 10529-010-0221-0$

Hutmacher, D.W., M. Sittinger and M.V. Risbud, 2004. Scaffold-based tissue engineering: Rationale for computer-aided design and solid free-form fabrication systems. Trends Biotechnol., 22: 354-362. DOI: 10.1016/j.tibtech.2004.05.005

Jamal, M., S.S. Kadam, R. Xiao, F. Jivan and T.M., Fernandes et al., 2013. Bio-origami hydrogel scaffolds composed of photocrosslinked PEG bilayers. Adv. Healthcare Mater., 2: 1142-1150. DOI: 10.1002/adhm.201200458

John, M., P. Albert, O. Andrew and J. Aaron, 1977. A simplified method for production and growth of multicellular tumor spheroids. Cancer Res., 37: 3639-3643.

Kruth, J.P., M.C. Leu and T. Nakagawa, 1998. Progress in additive manufacturing and rapid prototyping. CIRP Annals Manufact. Technol., 47: 525-540. DOI: 10.1016/S0007-8506(07)63240-5

Lee, J.M. and W.Y. Yeong, 2016. Design and printing strategies in 3D bioprinting of cell-hydrogels: A review. Adv. Healthcare Mater.

DOI: $10.1002 / \mathrm{adhm} .201600435$

Lee, J.M., M. Zhang and Y.W. Yeong, 2016. Characterization and evaluation of $3 \mathrm{D}$ printed microfluidic chip for cell processing. Microfluidics Nanofluid., 20: 1-15.

DOI: $10.1007 / \mathrm{s} 10404-015-1688-8$

Lutolf, M., P. Gilbert and H. Blau, 2009. Designing materials to direct stem-cell fate. Nature, 462: 433441. DOI: $10.1038 /$ nature 08602

Malda, J., J. Visser, F.P. Melchels, T. Jungst and W.E. Hennink et al., 2013. 25th anniversary article: Engineering hydrogels for biofabrication. Adv. Mater., 25: 5011-5028.

DOI: $10.1002 / \mathrm{adma} .201302042$
Mironov, V., T. Boland, T. Trusk, G. Forgacs and R.R. Markwald, 2003. Organ printing: Computeraided jet-based 3D tissue engineering. Trends Biotechnol., 21: 157-161. DOI: $10.1016 / \mathrm{S} 0167-7799(03) 00033-7$

Murphy, S.V. and A. Atala, 2014. 3D bioprinting of tissues and organs. Nature Biotechnol., 32: 773-785. DOI: $10.1038 /$ nbt.2958

Odde, D.J. and M.J. Renn, 2000. Laser-guided direct writing of living cells. Biotechnol. Bioeng., 67: 312-318.

DOI: $10.1002 /(\mathrm{SICI}) 1097-$ 0290(20000205)67:3<312::AID-BIT7>3.0.CO;2-F

Pati, F., J. Jang, D.H. Ha, S. Won Kim and J.W. Rhie et al., 2014. Printing three-dimensional tissue analogues with decellularized extracellular matrix bio-ink. Nat. Commun., 5: 1-11.

DOI: $10.1038 /$ ncomms4935

Patterson, J., M. Martino and A. Hubbell, 2010. Biomimetic materials in tissue engineering. Mater. Today, 13: 14-22. DOI: 10.1016/S1369-7021(10)70013-4

Peltola, S.M., F.P. Melchels, D.W. Grijpma and M. Kellomaki, 2008. A review of rapid prototyping techniques for tissue engineering purposes. Ann. Med., 40: 268-80.

DOI: $10.1080 / 07853890701881788$

Savas, T. and D. Utkan, 2013. Bioprinting for stem cell research. Trends Biotechnol., 31: 10-19. DOI: $10.1016 /$ j.tibtech.2012.10.005

Schiele, N.R., D.T. Corr, Y. Huang, N.A. Raof and Y. Xie et al., 2010. Laser-based direct-write techniques for cell printing. Biofabrication, 2: 032001-032001.

DOI: $10.1088 / 1758-5082 / 2 / 3 / 032001$

Schuurman, W., V. Khristov, M.W. Pot, P.R. Van Weeren and W.J.A. Dhert et al., 2011. Bioprinting of hybrid tissue constructs with tailorable mechanical properties. Biofabrication, 3: 021001-021001. DOI: 10.1088/1758-5082/3/2/021001

Seol, Y.J., T.Y. Kang and D.W. Cho, 2012. Solid freeform fabrication technology applied to tissue engineering with various biomaterials. Soft Matter, 8: 1730-1735. DOI: $10.1039 / \mathrm{C} 1 \mathrm{SM} 06863 \mathrm{~F}$

Spiller, K., S. Maher and A. Lowman, 2011. Hydrogels for the repair of articular cartilage defects. Tissue Eng., Part B, 17: 281-299. DOI: $10.1089 /$ ten.TEB.2011.0077

Wang, S., J.M. Lee and W.Y. Yeong, 2015. Smart hydrogels for 3D bioprinting. Int. J. Bioprint., 1: 3-14. DOI: 10.18063/IJB.2015.01.005 
Xu, T., W.X. Zhao, J.M. Zhu, M.Z. Albanna and J.J. Yoo et al., 2013. Complex heterogeneous tissue constructs containing multiple cell types prepared by inkjet printing technology. Biomaterials, 34: 130-139. DOI: 10.1016/j.biomaterials.2012.09.035

Yeong, W.Y. and C.K. Chua, 2013. Implementing additive manufacturing for medical devices: A quality perspective. Proceedings of the 6th International Conference on Advanced Research in Virtual and Rapid Prototyping, Oct. 1-5, Taylor and Francis Group, Portugal, London, pp: 115-120.
Yeong, W.Y., C.K. Chua, K.F. Leong and M. Chandrasekaran, 2004. Rapid prototyping in tissue engineering: challenges and potential. Trends Biotechnol., 22: 643-652.

DOI: 10.1016/j.tibtech.2004.10.004 